\title{
Research on the Construction of Rural Left-behind Children's Sports Assistance System in Guizhou Province*
}

\author{
Guanghui Yao \\ Physical Education Department \\ Liupanshui Normal University \\ Liupanshui, Guizhou, China
}

\author{
Long Zhang** \\ Scientific Research Department \\ Liupanshui Normal University \\ Liupanshui, Guizhou, China \\ **Corresponding Author
}

\begin{abstract}
Through literature research, interview and investigation, logical analysis, we have studied the sports assistance system for rural left-behind children in Guizhou Province. The study concludes that it is necessary for Guizhou to build a sports assistance system for left-behind children. Combining the research status of left-behind children in rural areas and field interview results, we should establish a "one main body, four platforms" assistance system which is based on the physical and mental health development of left-behind children, assisted by four platforms including government sports department, schools, families and the society, to provide a reference for the physical and mental health development of leftbehind children in rural areas.
\end{abstract}

Keywords-Guizhou; left-behind children; sports; assistance system

\section{INTRODUCTION}

With the appearance of the "migrant workers boom" during the social transition and urbanization in the early 1980s, the "left-behind children"

group also produced. Left-behind children as an extremely weak and growing social group, they are away from their parents' caring, supported by their grandparents or other relatives and friends, do not have a complete family and are difficult to accept good family education. According to statistics from the Ministry of Education, the number of rural left-behind children at the national compulsory education level in 2014 was 20.754 million. There were more left-behind children especially in Midwest regions such as Henan, Hunan, Sichuan, Anhui, Jiangxi and Guizhou. The education of leftbehind children has become an important problem in all social issues.

According to research data, Premier Li Keqiang made the "tragedy should not be repeated" instructions in 2015 to "the death of 4 left-behind children" event in Bijie, Guizhou Province; at the same time, some experts and scholars mentioned "psychological care for left-behind children should be highly valued", "the systematic family child protection

*Fund Project: Humanities and Social Sciences Research Project in University of Guizhou Provincial Department of Education (2016qn61). mechanism has not yet been established", "improvement of legal protection system for minors is a top priority", and "the government should strengthen the management and protection of rural left-behind migrant children" these viewpoints, which highly aroused the attention of all sectors of society to leftbehind children.

By combing the research results of left-behind children in the past 20 years, the study mainly focused on the current situation, mental health, personality traits, family education, school education, etc. of the children, while there were few results in the field of sports. Therefore, this research mainly focuses on the sports education of left-behind children, and takes the construction of Guizhou rural left-behind children's sports assistance system as a breakthrough, making the sports assistance for rural left-behind children in Guizhou province an important solution to the social problem of left-behind children. Provide practical reference ways for the healthy growth of left-behind children.

\section{THE NECESSITY OF CONSTRUCTING THE SPORTS ASSISTANCE SYSTEM FOR LEFT-BEHIND CHILDREN IN GUIZHOU}

\section{A. Implement Policies and Regulations to Ensure the Rights of Children Left Behind.}

Following the "Opinions on Strengthening Care and Education for Rural Left-behind Children at the Stage of Compulsory Education" of the Ministry of Education the "National Medium and Long-term Education Reform and Development Plan Outline (2010-2020)" of the Ministry of Education and "Chinese Children Development Outline (20112020)" of the National Woman Federation in 2013, Guizhou Province issued the "Notice on Guizhou Province Accurate Care Plan for Left-behind Children's Education(2015-2020)" (Planning and Development of Guizhou Education(2015) No. 255) and "Notice on Further Strengthening the Implementation Plan for Care and Assistance Protection of Children Leftbehind and in Plight" (Development of Guizhou Basic Education [2015] No. 251) in 2015 to strengthen the care and assistance protection of children left-behind and in plight. 
Comrade Xi Jinping, the President of the State, mentioned in the inspection of Guizhou Province "We must care for leftbehind children, improve working mechanism and measures and strengthen management and service". This provides reliable policy support to ensure good care services for leftbehind children in rural areas, the accurate care and support for left-behind children staying in school and the improvement of their happiness index.

\section{B. Improve the Education Assistance System and Build A Sports Assistance System.}

As an important part of the social assistance system, the assistance of left-behind children's education has always been concerned by people from all walks of life. However, the social phenomenon of left-behind children "behaving irregularly, lacking of affection, living helpless, and studying without teaching" still occurs. Left-behind children have social problems such as mental health, safety awareness, nutrition and health, distortion of values, and outstanding deviant behaviors. Left-behind children's sports education, as an important part of the education of left-behind children, when facing a series of social problems of left-behind children, should establish a good four-dimensional health view for leftbehind children, including physical health, mental health, moral health, and social adaptability. Implement the effect of sports education in promoting the physical, psychological, moral, and social adaptability of left-behind children.

\section{Respond to the Development Strategy of A Healthy City and Give Full Play to the Means of Physical Education.}

The problem of left-behind children is not only strongly supported by national policies, but also discussed theoretically and practically by academics from all walks of life. At the same time, in order to realize the goal of becoming a sports power country and the construction of a healthy city in Guizhou, the emphasis on youth sports has been continuously strengthened. The continuous improvement of the national fitness service system has accelerated the pace of youth sports development. Therefore, paying attention to left-behind children from the perspective of sports is not only a requirement for the healthy development of youth sports, but also a major solution to the social problems of left-behind children. So sports will become an important part in the healthy growth of left-behind children.

\section{REVIEW THE STUDY OF LEFT-BEHIND CHILDREN'S SPORTS ASSISTANCE}

We have sorted out the research achievements of leftbehind children at home and abroad in the field of sports. In foreign countries, because the family structure, lifestyle, way of thinking and cultural traditions are different from those in China, and laws and regulations are relatively sound to protect left-behind children, there are fewer left-behind children and less research on them. In China, the study of left-behind children in the sports field focuses on the current status of leftbehind children's physical exercise, the psychological influence of sports on left-behind children, and physical health interventions. The specific expression is: "Physical education is the important means to promote the psychological development and improve psychological quality of left-behind children, obtaining good results."; "physical exercise plays a role in physiological and biochemical effects on left-behind children"; "exercise is positively related to age, and with the increasing of age, physical activity can promote mental health development "; "Sports activities enable left-behind children to achieve a sense of accomplishment, turn attention away from anxiety and frustration, contribute to mental health, and improve social communication skills.""The purpose of leftbehind children to participate in sports activities is to enrich their extra-curricular life, strengthen physical health, and get relaxation"; "left-behind children are in a period of puberty, physical activity can improve their physical fitness and resistance";"In the physical education process, we pay attention to the differences in the physical conditions and personality of left-behind children, use games, competitions and other teaching methods to let left-behind children participate in collective activities"; Sports games can promote physical and mental development of left-behind children in rural areas, cultivate optimistic attitude, and improve social adaptability.""Establishing a sports assistance system will promote the physical and psychological development of leftbehind children, strengthen the cultivation of sports reserve talents, protect traditional ethnic sports, and expand the number of sports persons".

In summary, left-behind children have existed in China for more than 20 years. Although researchers have studied the problem of left-behind children for nearly 20 years, the explanation on the sports effect on left-behind children is not systematic enough. The domestic legislation on left-behind children is not sound. The definition of left-behind children is not clear and the age range is not clear. Lack of family education directly leads to left-behind children having low physical and mental health. Research on left-behind children mainly focuses on the status quo and mentality of them. As for health, personality traits, family education and school education, although holding extracurricular sports activities is an effective mean to promote the mental health of left-behind children, there is little research on physical education as an assistance system for left-behind children. Research on leftbehind children in the field of sports should be conducted more scientifically and systematically in the future, providing practical reference for the physical and mental health development of left-behind children.

\section{CONSTRUCTION OF LEFT-BeHIND CHILDREN SPORTS ASSISTANCE SYSTEM IN GUIZHOU}

In the process of constructing the rural sports assistance system for left-behind children in Guizhou Province, combined with the results of interviews and investigations of rural leftbehind children in Guizhou, it is necessary to establish the "one main body and four platforms" assistance system which is based on the physical and mental health development of leftbehind children, assisted by four platforms including government sports department, schools, families and the society. The establishment of a sports assistance platform for left-behind children in the absence of family education requires the integration of various resources of the government, schools, families, and society. The formation of the operation, 
management, and guarantee systems for left-behind children's sports assistance system requires the government's attention, legal and policy guarantees, and capital investment to further promote the effective implementation of the care service program for rural left-behind children in Guizhou Province and increase the happiness index of them.

\section{A. Construction Principles}

\section{1) The principle of science}

The construction of a rural sports assistance system for leftbehind children in Guizhou Province must clearly reflect the relation between the sports needs and the various aid platforms. The establishment of left-behind children's sports needs should be based on practical investigations, closely follow the policies of care and service for left-behind children in Guizhou Province, incorporate the social problems of rural left-behind children such as mental health, safety issues, behavioral disorders, and values distortions. And can be effectively applied to the practice of sports assistance service for rural leftbehind children in Guizhou Province. It is a manifestation of the principle of science.

\section{2) The principle of region}

From the perspective of region, with the physical and mental health development of left-behind children as the main body, the government' s sports department, schools, families, and society as four assistance platforms, carry out the overall construction of the system. Regional characteristic of Guizhou should be considered in the process of practice, practically apply it to the sports aid system.

\section{3) The principle of equalization}

Equalization is the goal of the current government and social public service career. The building of sports assistance system mainly focuses on providing public sports resources. That is supported by the government's investment in sports finance. The principle of equalization emphasized here is saying that making left-behind children in different regions in Guizhou have equal satisfaction level with sports assistance services on the premise of economic, political and cultural differences.

\section{B. The Path of Construction}

The construction of the sports assistance system for rural left-behind children in Guizhou makes sports one of the means to solve the problem of left-behind children's education and can ensure the healthy growth of them. The physical education content in school can be set according to the characteristics of the region, which can protect and pass on the national characteristic sports forms and play an important role in sustainable development. Through correct guidance, more children can be involved in sports to ensure the reserve force for sports; through the construction and promotion of sports assistance system, it is possible to expand sports population and promote social health development. Through the construction of the sports assistance system for rural leftbehind children in Guizhou, we can integrate the strengths of all levels of society from the perspective of sports, to focus on the healthy growth of left-behind children, and propose the "one main body and four platforms" sports assistance system.

\section{1) Focus on the physical and mental health of left-behind} children

In order to promote the physical and mental health of leftbehind children in rural areas in Guizhou, all physical and mental health needs of left-behind children should be put in the first place. Through the platform forces of the government, society, schools, and families, integrate sports resources at all levels of society and implement sports intervention for leftbehind children, and change the sports status of left-behind children from intervened to spontaneous. We can establish a

"Left-behind Children's Physical and Mental Healthy Growth Personal Profile", and set up a left-behind child's sports lifestyle exercise schedule, which mainly includes exercise programs, frequency, time, intensity, and so on. In school, they are mainly collected monthly by teachers to make the e-files, while they are collected by the leader of an interest group outside the school.

\section{2) Government sports department assistance platform}

The government sports department assistance platform mainly provides assistance from the perspective of the integration of sports resources. In terms of laws and policies, basing on the Opinions on Strengthening Care and Education for Rural Left-behind Children at the Stage of Compulsory Education, the Outline of the National Medium- and Longterm Education Reform and Development Plan (2010-2020), the Outline of Chinese Children's Development (2011- 2020), "Notice on Program of Precision Care for the Left-behind Children's Education in Guizhou (2015-2020) "and "Notice on Further Implementing the Program of Care and Assistance Protection of Children Left Behind and in Difficulties ", all regions should implement the rules and policy content for leftbehind children according to local conditions. In academic research, the study of rural left-behind children will be included in the topics selected for social science research. Actively explore intervention experiments, training mechanisms, and innovative models for the physical and mental health development of left-behind children. For example, formulate relevant measures for the "left-behind children's sports assistance system" and implement them to left-behind children; In terms of fund guaranteeing, we will provide necessary policies for left-behind children in rural areas. We will set up a special fund for left-behind children's sports development based on the local fiscal revenue. We will do things for the children using the fund such as improving school sports facilities and constructing the team of sports teachers in rural area.

\section{3) Social sports assistance platform}

The social sports assistance platform mainly cares for leftbehind children from the perspectives of funding, helping, and succoring. On the one hand, we make full use of social charities, carry out charitable sports activities and sports donation activities for left-behind children, so as to raise donations for them, and provide materialistic support for the sports and cultural life of them. On the other hand, capable social enterprises can sponsor left-behind children's events and sports games to let them participate in sports activities. It not only allows them to exercise physically and mentally, develops 
their sports interest in a good atmosphere, but also discovers their sports potential, reserves sports talents, and broadens the input path for sports talents.

\section{4) School sports assistance platform}

At present, school education pays most attention to students' academic performance, and it pays less attention to students' safety, physical fitness and mental health. The physical condition of young students is not optimistic. This has aroused school's and even national attention to sports. Leftbehind children are disadvantaged groups in school. Sports should play its educational function. Schools should build a platform to promote the harmonious development of leftbehind children's physical and mental health, that is, establish a school sports assistance platform. For example, in terms of teaching, schools can combine the geographical and school characteristics to develop sports activities in accordance with the requirements of the new curriculum standards. With respect to ethnic characteristics, we can add martial arts, dragon dance, lion dancing, hand-dance and other ethnic elements. In the mental health curriculum, sports should be used as an important mean to interfere with physical and mental problems of left-behind children. In short, while enriching the content of physical education courses, we should carry out teaching work in light of the physical and mental health of left-behind children in rural areas. As for school physical education teacher team construction, we should use national training, special and particular post internships, free teacher education program, etc. Specifically we can let college physical education students go to primary and secondary schools for internships and teaching support. So that can increase the number of rural physical education teachers and improve the rural physical education teachers' professional level. In terms of sports ideology propaganda, schools should focus on the sports health view of "health comes the first", and actively promote the importance of physical exercise. Specifically, we can adopt ways of electronic media propaganda, broadcasting and fixed-site publicity. At the same time, schools should also establish physical records for students' physical conditions. It is better to form a physical test station for left-behind children in schools and constantly pay attention to the physical health status in order to improve rural physical education contents.

\section{5) Family sports assistance platform}

Family sports are not only the supplement and continuation of school sports, but also the front line of school sports activities. However, the guardians of left-behind children have dependence on schools, shirk their duties and put the responsibility of educating children completely to school. However, the lack of family education will inevitably lead to the lack of family sports training and education for the leftbehind children. Therefore, we must establish a guardian's view of education, health, sports and talents first. Let the families of left-behind children attach importance to cultivating their physical exercise. Specifically, you can participate in sports activities, sports training and sports exchanges. Participating in physical exercise is an effective way to form a family sports aid platform for left-behind children.

\section{CONCLUSION}

This study proposes the necessity of constructing a rural sports assistance system for left-behind children in Guizhou Province. Based on the research status of rural left-behind children and the results of on-site interviews, it is proposed that the rural sports assistance system should be established into a "one main body, four platforms" system with "the development of left-behind children's physical and mental health as the main body, government sports department assistance, school sports assistance, family sports assistance and social sports assistance as four platforms". The operation, management, and protection of left-behind children's sports assistance system require government attention, legal and policy guarantees and capital investment. We must promote the effective implementation of the Guizhou Rural Left-behind Children Care Service Plan and improve the left-behind children's happiness index.

\section{REFERENCES}

[1] Nie Mao, Li Huajun,Sad about villages - Anxious thoughts about children left behind in rural China[M]. Beijing: People's Daily Press, 2008

[2] Sun Lei,Study on the Influence and Effects of Extracurricular Sports Activities on the Mental Health of Left-behind Children in Rural Junior High Schools - - Taking Daming County of Handan City as an Example[D].Hebei Normal University Master Thesis, 2014

[3] Tang Lijun, Pan Shaowei, Wang Jianmin et al., Analysis of the Role of Sports in Building a Harmonious Society - - Analysis from the Perspective of Sports Function[J] Sports Science, 2008,29(2),pp.45-47.

[4] Wang Qiyan. Research on the construction of urban and rural social assistance system in China[M]. Beijing: People's Publishing House, 2009

[5] Wang Mengqin. Research on the needs and social support of rural leftbehind children[D]. Southwest University, 2012.

[6] Wang Qian, Wang Xuefeng. Effects of physical education teaching on mental health of left-behind children $[\mathrm{J}]$ Contemporary Education Theory and Practice, 2010, 2(5),pp.11-12.

[7] Chen Zhijun. Preliminary study on physical exercise to promote the development of psychological health of left-behind children in rural areas [J]. Huazhang, July,2011.

[8] Yang Rong, Research on the Influence of Sports Activities on the Behavior of Left-behind Children in Rural Areas[J]. Journal of Chizhou University, 2013, 27(2), pp.16-18.

[9] Kong Boshuang, Li Jianghua, Ding Weixiang, et al. Study on psychological sub-health and sports education countermeasures for leftbehind children in Jiangxi Province[J] Science and Technology Innovation Guide, 2010, 25,pp.140-142.

[10] Gao Jianlei, Investigation on Extracurricular Physical Training Characteristics of Left-behind Children in Rural Areas: A Case Study of Undeveloped Areas in Western Guangdong Province[J].Journal of Guangzhou Physical Education Institute,2009,29(1),pp.9-13.

[11] Chen Qiaodi, Zhao Ruili.Exploration of the Function of Physical Education in Primary and Secondary Schools in Promoting the Physical and Mental Development of Rural Left-behind Children[J].Journal of Tianshui Normal University,2009,29(5),pp.105-106.

[12] Pan Hongxia, Chu Qinggui, An analysis of the role of sports in promoting the healthy growth of rural left-behind children[J]. Journal of Chifeng University(Natural Science Edition),2013,29(10),pp.160-161.

[13] Chu Qinggui, Pan Hongxia, Research on the role of sports games in the physical and mental development of left-behind children in rural areas[J]. Journal of Chifeng University(Natural Science Edition), 2013, 29(11),pp.189-191. 
[14] Liu Xuejian,Chen Xi.Thinking on the Necessity of Establishing the "Sports Assistance System" for Rural Left-behind Children --- Based on the Enlightenment of Venezuela's "Music Assistance System"[J]. Bulletin of Sport Science and Technology, 2016,24(2),pp.137-139. 